\title{
Rituximab-induced serum sickness and anaphylaxis in a child with nephrotic syndrome
}

\author{
Meral Torun Bayram, Alper Soylu ${ }^{\oplus}$ Salih Kavukçu ${ }^{\odot}$ \\ Department of Pediatrics, Dokuz Eylul University Faculty of Medicine, İmir, Turkey.
}

\begin{abstract}
Background. Rituximab is effective for treatment of children with refractory nephrotic syndrome (NS). However, the drug may cause serum sickness characterized by fever, rash, and arthralgia 10-14 days after primary antigen exposure or within a few days after secondary antigen exposure. Rituximab may also lead to anaphylaxis. It is important to recognize rituximab-induced serum sickness (RISS) clinically, as it may mimic various infectious or vasculitic diseases.
\end{abstract}

Case. A six-year-old male with NS treated with rituximab presented with diffuse arthralgia and myalgia eight days after the first dose. He developed an urticarial rash and arthralgia one week after the second dose, while he had swelling of lips and periorbital regions, choking sensation and erythematous rash in whole body within minutes after the third dose of rituximab. The first two reactions resemble typical serum sickness whereas the third reaction seem to be an anaphylaxis/anaphylactoid reaction.

Conclusions. Although rituximab-induced serum sickness is typically self-limited, further infusions of rituximab should be avoided as it may provoke more severe symptoms. Most of the previous reported cases of RISS are patients with autoimmune or hematologic disorders. We present the first pediatric case with membranous nephropathy and RISS. The patient also developed anaphylactoid reaction during the third rituximab infusion.

Key words: rituximab, nephrotic syndrome, serum sickness, anaphylaxis.

Nephrotic syndrome (NS) is a renal disease characterized by massive proteinuria, hypoproteinemia and generalized edema. In NS, first line treatment is prednisolone. However, other treatments such as cyclophosphamide and cyclosporine A are used in frequently relapsing or steroid-resistant NS, respectively. Recently, rituximab has been used in both forms of NS when the response to other treatment options is inadequate. ${ }^{1}$

$\bigotimes \quad$ Meral Torun Bayram

meralt.bayram@yahoo.com.tr

Received 8th October 2019, revised 2rd January 2020, 12th March 2020, accepted 20th March 2020.

Abstract was presented in 51st congress of European Society for Paediatric Nephrology which was held in Antalya, Turkey (3-6 October 2018).
Rituximab is a chimeric murine/human antiCD20 monoclonal antibody binding to the B-cell surface antigen CD20. Although its mechanism of action remains unclear, rituximab is effective for preventing relapse in refractory NS while avoiding steroid side effects.,3 On the other hand, rituximab has adverse events such as fever, cough, dyspnea, and erythema in patients with NS.4-6 Rituximab may also rarely induce serum sickness. ${ }^{7}$

Rituximab-induced serum sickness (RISS) is a rare type III hypersensitivity reaction which occurs after injecting foreign antigens and is characterized by fever, rash, and arthralgia. RISS is relatively common among patients who have autoimmune and hematologic diseases. So far, only two cases of RISS in patients with NS (17 and 50-years-old) have been reported..$^{8,9} \mathrm{IgE}$ mediated hypersensitivity reactions, confirmed by positive skin tests, have also been reported. ${ }^{10}$ 


\section{Case Report}

A 6-year-old boy was diagnosed with NS at 2 years of age. Renal biopsy performed for steroid resistant NS revealed membranous nephropathy. Evaluation for secondary causes of membranous nephropathy (HBV, HCV, HIV, syphilis serologies; ANA, anti-dsDNA, C3, C4) was inconclusive. As the parents did not accept cyclophosphamide treatment, calcineurin inhibitor was instituted. He responded to steroid plus cyclosporine A, but experienced frequent relapses. Thus, rituximab treatment was instituted as $375 \mathrm{mg} / \mathrm{m}^{2}$ intravenously over 4 hours. He was premedicated with chlorphenoxamine $5 \mathrm{mg}$ intravenously (IV) and acetaminophen $10 \mathrm{mg} / \mathrm{kg}$ IV. Trimethoprimsulfamethoxazole $\left(150 \mathrm{mg} / \mathrm{m}^{2} /\right.$ day in 3 divided doses) was started for Pneumocystis jirovecii pneumonia prophylaxis. He had no infusion reactions. Eight days after the first dose, the patient presented with diffuse incapacitating arthralgia and myalgia, and he was hospitalized. Physical examination and laboratory tests were normal at that time. His symptoms improved within 24 hours without any intervention.

Two months later, the second rituximab infusion (in the same manner as the previous dose with premedication) was given as the number of CD19 and CD20-positive cells increased. The patient presented with diffuse rash and arthralgia the following week (Fig. 1). This time, his symptoms resolved spontaneously within two days.

Three weeks later, during the third cycle of rituximab (with the same previous dosing schedule), he developed swelling of lips and periorbital regions, choking sensation, generalized erythematous rash during rituximab infusion leading to discontinuation of the infusion (Figs. 2-4). On physical examination, the patient had a mild fever (37.8 ${ }^{\circ} \mathrm{C}$ ) and was hemodynamically stable with a pulse rate $96 / \mathrm{min}$, respiratory rate of $22 / \mathrm{min}$, and blood pressure 105/65 $\mathrm{mmHg}$. There was no sign of acute infection. He was treated with intramuscular methylprednisolone and IV adrenaline with rapid resolution of symptoms.
His laboratory tests were as follows: leukocyte $5000 / \mathrm{mm}^{3}$ with $43 \%$ neutrophils and $3.5 \%$ eosinophils, erythrocyte $4.4 \times 10^{6} / \mathrm{mm}^{3}$, platelet $347 \times 10^{3} / \mathrm{mm}^{3}$, C-reactive protein $0.7 \mathrm{mg} / \mathrm{L}$, Anti-nuclear antibody negative, IgG $1102 \mathrm{mg} /$ dl (N:700-1600), IgM 108 mg/dl (N:20-200), IgA $147 \mathrm{mg} / \mathrm{dl}$ (N:70-400), IgE 147 IU/ml (N:0-52), C3 116 mg/dl (N: 81-157), C4 13.9 mg/dl (N:1339). Urinalysis and viral serology were normal.

The symptoms and signs of the patient were attributed to RISS (the first and second infusions) and to anaphylaxis (third infusion) based on the temporal relation of the clinical findings with rituximab infusion and rapid clinical improvement. Thus, rituximab treatment was discontinued.

Informed written consent was obtained from the parents for reporting the case.

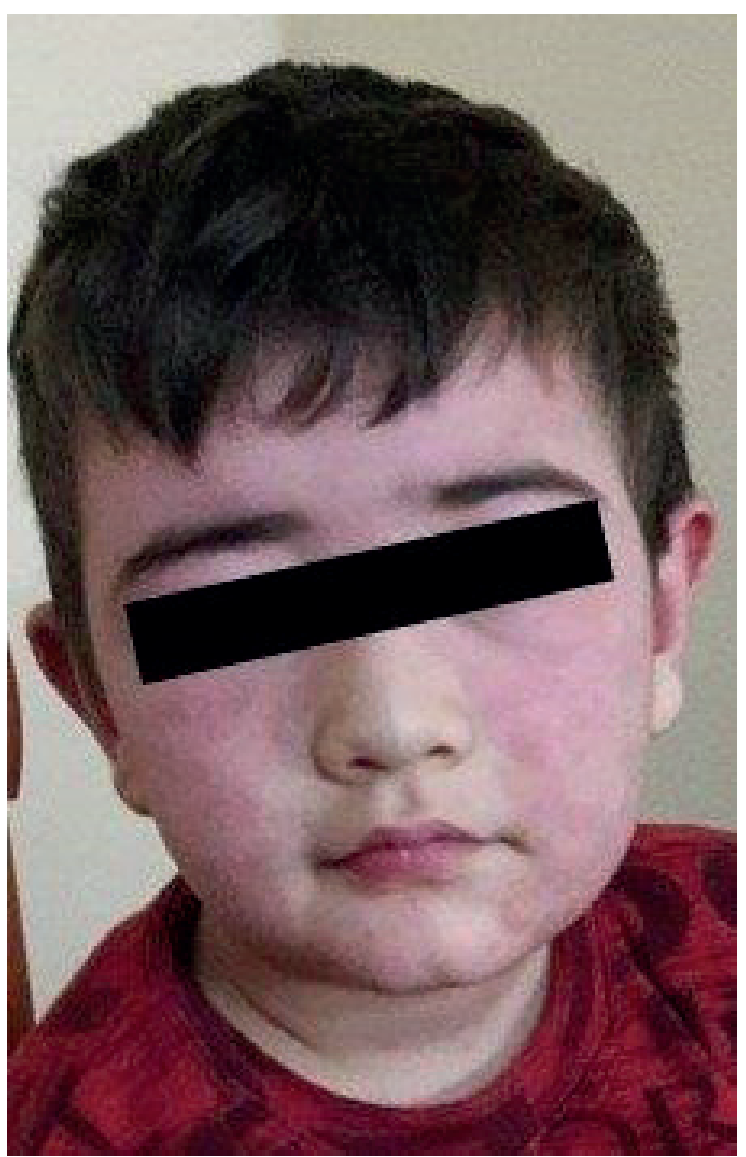

Fig. 1. The rash associated with the second dose of rituximab. 

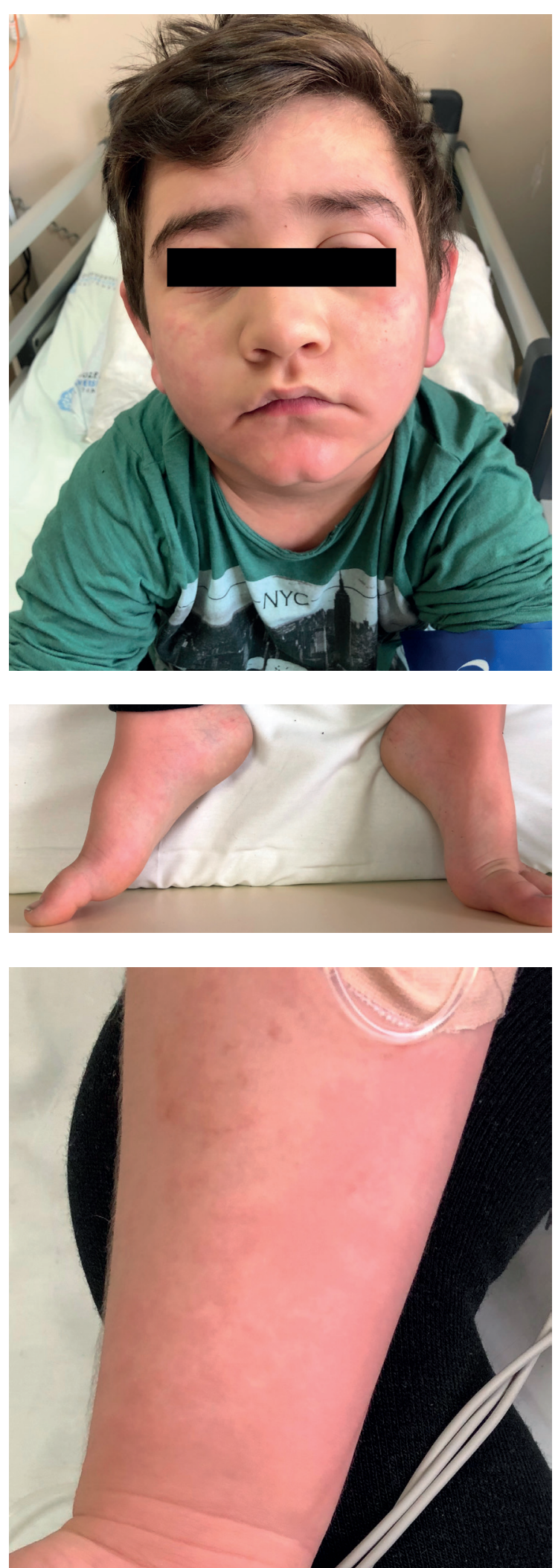

Figs. 2-4. The rash associated with the third dose of rituximab on the face, feet and forearm.

\section{Discussion}

Rituximab is a chimeric anti-CD20 mousehuman monoclonal antibody. Once bound to CD20, rituximab eliminates B cells by direct, complement and antibody-dependent cellular cytotoxicity. ${ }^{11}$ Rituximab has been generally used to treat various autoimmune diseases and hematological malignancies in which $B$ cells or CD20-bearing cells are participants. ${ }^{12,13}$ While it is frequently licensed for these diseases, it is not yet licensed for nephrotic syndrome in many countries.

Rituximab reactions mostly occur with the first infusion. These are associated with general infusion reactions, including fever, chills, and rigors. However, it may also be allergic/ anaphylactoid spectrum reactions such as urticaria, angioedema and hypotension. ${ }^{14}$ Simple reactions are thought to be due to a cytokine release syndrome potentially triggered by the murine element of the antibody. ${ }^{14}$

RISS was first reported in 2001 in a patient treated with rituximab for refractory autoimmune polyneuropathy. Since then, about 50 cases of RISS have been published in patients with rheumatological and hematological diseases. ${ }^{14,15}$ However, there are only two case reports of RISS associated with NS, one being a 17-yearold girl with refractory steroid-dependent NS and the other was a 50-year-old female with membranous nephropathy. ${ }^{8,9}$ Nevertheless, to the best of our knowledge, no pediatric case with RISS has yet been reported in membranous nephropathy.

RISS usually occurs 10-14 days after primary antigen exposure or within a few days of secondary antigen exposure. Clinical findings include fever, arthralgia, urticarial rash, lymphadenopathy, proteinuria and gastrointestinal symptoms. ${ }^{14}$ It may also be accompanied by elevated inflammatory markers, high immunoglobulin and decreased complement levels.

The mechanism of RISS is not exactly known, but it is considered that B-cells sensitized to 
rituximab are lysed, resulting in the release of antibodies to rituximab and formation of immune complexes. ${ }^{16}$ After that, formation of these immune complexes result in immune complex-mediated type III hypersensitivity, resulting in complement activation and mast cell degranulation. It is considered that human anti-chimeric antibodies (HACAs) may be associated with RISS and HACAs development may provide important clues to diagnosing serum sickness. However, not all of the patients with RISS developed HACAs in previous studies. ${ }^{14}$ Furthermore, absence or presence of HACAs has not been consistently associated with RISS development. ${ }^{17}$ Therefore, the diagnosis of RISS should be made based on history and symptoms. ${ }^{14}$ Our patient presented with diffuse arthralgia and myalgia eight days after the first rituximab dose. He developed an urticarial rash and arthralgia one week after the second rituximab dose, while he had swelling of lips and periorbital regions, choking sensation and erythematous rash in whole body within minutes after the third dose of rituximab. It is important to recognize the temporal relationship of symptoms with the dose of rituximab infusion (usually 7 days), as RISS is a clinical diagnosis. ${ }^{14}$ The first two reactions resembled typical serum sickness with regard to both the clinical findings and the temporal relation to infusion. However, the third reaction that developed during infusion of rituximab seemed to be an anaphylaxis/ anaphylactoid reaction clinically. IgE-mediated hypersensitivity reactions to rituximab have been reported even during $4^{\text {th }}$ or $5^{\text {th }}$ infusions previously. Positive skin test was reported in half of the cases. ${ }^{10}$ We could not measure serum HACAs level and the RISS diagnoses was made according to the history and symptoms. We also did not attempt to perform a skin test for confirmation of hypersensitivity to rituximab.

RISS has been reported at a higher rate in patients with autoimmune diseases compared to those with lymphoma and NS. ${ }^{4}$ Thus, it has been proposed that rituximab may be more immunogenic in autoimmune diseases due to the highly activated B lymphocytes. ${ }^{18}$

Steroid treatment is the mainstay of management in RISS. ${ }^{8,14}$ Antihistaminics may also provide extra benefit. A systemic review showed that symptoms resolve in $2.15 \pm 1.34$ days. ${ }^{14}$ Rituximab is usually discontinued in patients who developed RISS. On the other hand, rituximab was continued in four RISS cases along with steroid therapy in a previous study..$^{14}$ We also used intramuscular methylprednisolone with adrenaline and the next day the reaction subsided almost completely. However, we did not continue rituximab therapy in our patient.

In conclusion, RISS is often a benign condition and usually resolves with steroid therapy. However, it is important to recognize and distinguish RISS clinically from the other side effects of rituximab. If RISS is not recognized and the patient is re-exposed to rituximab, more serious clinically consequences may develop.

\section{REFERENCES}

1. Kamei K, Ogura M, Sato M, Sako M, Iijima K, Ito $\mathrm{S}$. Risk factors for relapse and long-term outcome in steroid-dependent nephrotic syndrome treated with rituximab. Pediatr Nephrol 2016; 31: 89-95.

2. Benz K, Dotsch J, Rascher W, Stachel D. Change of the course of steroid-dependent nephrotic syndrome after rituximab therapy. Pediatr Nephrol 2004; 19: 794-797.

3. Nozu K, Iijima K, Fujisawa M, Nakagawa A, Yoshikawa N, Matsuo M. Rituximab treatment for posttransplant lymphoproliferative disorder (PTLD) induces complete remission of recurrent nephrotic syndrome. Pediatr Nephrol 2005; 20: 1660-1663.

4. Kamei K, Ogura M, Sato M, Ito S, Ishikura K. Infusion reactions associated with rituximab treatment for childhood-onset complicated nephrotic syndrome. Pediatr Nephrol 2018; 33: 1013-1018.

5. Iijima K, Sako M, Nozu K, et al; Rituximab for Childhood-onset Refractory Nephrotic Syndrome (RCRNS) Study Group. Rituximab for childhoodonset, complicated, frequently relapsing nephrotic syndrome or steroid-dependent nephrotic syndrome: a multicentre, double-blind, randomised, placebo-controlled trial. Lancet 2014; 384: 1273-1281.

6. Ito S, Kamei K, Ogura M, et al. Survey of rituximab treatment for childhood-onset refractory nephrotic syndrome. Pediatr Nephrol 2013; 28: 257-264. 
7. Chung $\mathrm{CH}$. Managing premedications and the risk for reactions to infusional monoclonal antibody therapy. Oncologist 2008; 13: 725-732.

8. Maeda R, Kawasaki Y, Ohara S, Suyama K, Hosoya M. Serum sickness with refractory nephrotic syndrome following treatment with rituximab. CEN Case Rep 2018; 7: 69-72.

9. Cheong J, Ooi K. Rituximab-induced serum sickness in the treatment of idiopathic membranous nephropathy. Clin Kidney J 2018; 11: 51-53.

10. Soyer O, Demir S, Bilginer $\mathrm{Y}$, et al. Severe hypersensitivity reactions to biological drugs in children with rheumatic diseases. Pediatr Allergy Immunol 2019; 30: 833-840.

11. Randall KL. Rituximab in autoimmune diseases. Aust Prescr 2016; 39: 131-134.

12. Mok CC. Rituximab for the treatment of rheumatoid arthritis: an update. Drug Des Devel Ther 2013; 8: 87-100.

13. DeMonaco NA, Jacobs SA. Serum sickness in a patient with follicular lymphoma after rituximab and radioimmunotherapy with ibritumomab tiuxetan. Clin Nucl Med 2007; 32: 933-934.
14. Karmacharya P, Poudel DR, Pathak R, et al Rituximab-induced serum sickness: a systematic review. Semin Arthritis Rheum 2015; 45: 334-340.

15. Le Guenno G, Ruivard M, Charra L, Philippe P. Rituximab-induced serum sickness in refractory immune thrombocytopenic purpura. Intern Med J 2011; 41: 202-205.

16. Finger E, Scheinberg M. Development of serum sickness-like symptoms after rituximab infusion in two patients with severe hypergammaglobulinemia. J Clin Rheumatol 2007; 13: 94-95.

17. Goto S, Goto H, Tanoshima R, et al. Serum sickness with an elevated level of human anti-chimeric antibody following treatment with rituximab in a child with chronic immune thrombocytopenic purpura. Int J Hematol 2009; 89: 305-309.

18. Vendramin C, Thomas M, Westwood JP, McGuckin S, Scully M. Rituximab-induced acute and delayed serum sickness in thrombotic thrombocytopenic purpura: the role of anti-rituximab antibodies. Br J Haematol 2019; 184: 858-861. 